Check for updates

Cite this: RSC Adv., 2018, 8, 32565

Received 22nd July 2018

Accepted 13th September 2018

DOI: $10.1039 / \mathrm{c} 8 \mathrm{ra0} 6208 \mathrm{k}$

rsc.li/rsc-advances

\section{Reduced graphene oxide-supported methylene blue nanocomposite as a glucose oxidase-mimetic for electrochemical glucose sensing $\dagger$}

\begin{abstract}
Shaojun Yang, Daliang Liu, Qing Bo Meng, (D) Shuyao Wu* and Xi-Ming Song (D) *
In this paper, a hybrid nanocomposite (MB-rGO) was synthesized based on the $\pi-\pi$ stacking interactions between methylene blue (MB) and reduced graphene oxide ( $\mathrm{rGO}$ ). The as-synthesized nanocomposite was characterized by SEM, TEM, XRD, FTIR, UV-vis and XPS spectra. UV-vis spectroscopy and electrochemical tests suggested the MB-rGO modified on the electrode exhibited glucose oxidasemimetic catalytic activity towards glucose, and displayed excellent electrocatalytic performance for electrochemical detection of glucose with a wide linear range from 1.04 to $17.44 \mathrm{mM}$, a low detection limit of $45.8 \mu \mathrm{M}$ and a large sensitivity of $13.08 \mu \mathrm{A} \mathrm{mM} \mathrm{m}^{-1} \mathrm{~cm}^{-2}$. The proposed glucose sensor also showed high stability, reproducibility and good abilities of anti-interference to dopamine, ascorbic acid and uric acid. Moreover, the modified electrode was used to determine glucose concentration in human blood serum samples with satisfactory results.
\end{abstract}

\section{Introduction}

In recent years, with the gradual improvement of people's living standards and the aggravating trend of population aging, the incidence of diabetes is gradually increasing around the world, gravely threatening human health. ${ }^{1-3}$ Monitoring blood glucose levels is of vital importance for prevention, diagnosis and treatment of diabetic patients. ${ }^{4}$ The traditional methods of detecting glucose mainly include spectrophotometry, ${ }^{5,6}$ chromatography, ${ }^{7}$ and electrochemical methods. ${ }^{8,9}$ Thereinto, electrochemical methods have been widely used with the advantages of speed, accuracy, and low cost. ${ }^{10}$ Therefore, much more effort has been made to develop electrochemical glucose sensors with high sensitivity, excellent selectivity and good reliability for detecting glucose concentration in blood. ${ }^{11-13}$ Electrochemical glucose sensors are mainly classified into two types based on enzyme and enzyme-free (nonenzymatic) electrochemical sensors. ${ }^{14}$ The former, glucose oxidase (GOx)-based electrochemical sensors, commonly exhibits good sensitivity and selectivity, but its performance is badly influenced by complicated immobilization procedure, critical operational conditions and enzymatic chemical instability. ${ }^{15}$ Moreover, enzymatic electrochemical glucose sensors have the disadvantage of short shelf life, which resulting in high fabrication cost. $^{16,17}$ Hence, the development of nonenzymatic glucose

Liaoning Key Laboratory for Green Synthesis and Preparative Chemistry of Advanced Materials, College of Chemistry, Liaoning University, Shenyang 110036, China. E-mail: sywu@lnu.edu.cn; songlab@lnu.edu.cn; Tel: +86-24-62202378

$\dagger$ Electronic supplementary information (ESI) available. See DOI: $10.1039 / \mathrm{c} 8 \mathrm{ra} 06208 \mathrm{k}$ sensors becomes a attractive alternative. ${ }^{17}$ With the rapid development of nanotechnology, the great progress of enzymefree glucose sensors has been made. Numerous nanocatalysts such as metals, ${ }^{18,19}$ metal oxide ${ }^{20,21}$ and their hybrid with carbonbased nanomaterials ${ }^{13,22,23}$ have been explored for electrooxidation of glucose. Besides, inorganic complexes ${ }^{24,25}$ were also used for the electrocatalytic oxidation of glucose in nonenzymatic glucose sensors. Whereas a few of disadvantages still exist in the developed nonenzymatic sensors. The metals-based (particularly noble metals) nonenzymatic sensors often suffer from various of problems such as high cost, inferior stability caused by chloride ions triggered the poisoning phenomena, ${ }^{10,26}$ and lack of selectivity in the catalysis of glucose if some carbohydrates coexist. ${ }^{27}$ Therefore, novel electrode materials with simple, low-cost and good stability and selectivity, particularly organic materials without metals, are still desirable in development of nonenzymatic sensors. As far as we know, the redox-active functional organic molecules such as methylene blue (MB), which can initiate the electrochemical reactions and generate the change of electrical current as the enzyme-mimetic electrode materials, are rarely reported, owing to their relative poor conductivity. The electrochemical sensors based on the materials using the functional organic molecules as redox species are expected to have advantages of good reversibility and sensitive response signal through combining other conductive materials.

$\mathrm{MB}$, a typical water-soluble cationic organic dye full of $\pi$ electrons, has been used as a redox indicator for detecting some biomolecules by electrochemical method, for instance, Plasmodium falciparum histidine-rich protein 2 in immunosensor, ${ }^{28}$ miRNA in miRNA biosensor ${ }^{29}$ and DNA damage in 
electrochemical DNA-sensor. ${ }^{30}$ The formal potential of $\mathrm{MB}$ is within the scope of -0.10 to $-0.40 \mathrm{~V}$ (versus SCE) at $\mathrm{pH}$ range from 4 to 11 , which is near to the potential of common redox enzymes or proteins, such as glucose oxidase, ${ }^{9}$ horseradish peroxidase $^{31}$ and hemoglobin. ${ }^{32}$ Given the above facts, MB can be considered to play a role of redox enzyme and used to develop novel enzyme-free electrochemical sensors. To the best of our knowledge, $\mathrm{MB}$, as a mimetic enzyme, for constructing nonenzymatic electrochemical sensor has not been reported.

Because of its good water solubility and poor conductivity, MB can't be directly used on the electrode. But, the electron transfer ability and the stability of MB as electrode materials could be improved by compositing it with other functional materials. $^{33}$ Graphene and its composites can well promote electron transfer and have been extensively used as electrode materials owing to their unique structure and excellent properties. ${ }^{\mathbf{9} 3-37}$ In this work, a MB-functionalized reduced graphene oxide (MB-rGO) nanocomposite was fabricated through $\pi-\pi$ stacking interactions between MB and rGO. Due to the modification by $\mathrm{MB}$, rGO can keep its monolayer sheet structures in water and better conductivity. The MB-rGO/glassy carbon (GC) electrode constructed by the as-prepared nanocomposite (MBrGO) exhibited good electrochemical catalytic performance for glucose, and could detect glucose with wide linear range and high sensitivity, indicating the redox organic molecules could construct enzyme-mimetic electrochemical sensors by combining other functional materials.

\section{Experimental}

\subsection{Materials}

MB was purchased from Aladdin Reagent Co. Ltd. (China). Hydrazine hydrate (35 wt\%) was provided by Sigma chemical Co. Ltd. (China). Natural graphite power (99.95\%, 325 mesh), glucose and all other chemicals were obtained from Sinopharm Chemical Reagent Co. Ltd. (China). Human serum samples were taken from normal healthy volunteers at the Affiliated Hospital of China Medical University, and informed consent was obtained, respectively. All the experiments were conducted with the approval of the Ethics Committee of China Medical University. Unless otherwise stated, other reagents were of analytical grade and used as received. All solutions were prepared by using Milli-Q purified water $\left(>18.0 \mathrm{M} \Omega \mathrm{cm}^{-1}\right)$ sterilized at high temperature.

\subsection{Preparation of MB-rGO nanocomposite}

Graphene oxide (GO) was prepared from nature graphite powder according to the method of Hummers. ${ }^{33}$ The preparation of MB-rGO nanocomposite was synthesized following the procedures described in the literature. ${ }^{38}$ Briefly, $1.5 \mathrm{mg}$ of $\mathrm{MB}$ was dissolved in $15 \mathrm{~mL}$ water and then the solution was mixed with $20 \mathrm{~mL}$ of $\mathrm{GO}\left(0.1 \mathrm{mg} \mathrm{mL}^{-1}\right)$ aqueous dispersion under constant sonication. After adding $10 \mu \mathrm{L}$ of hydrazine hydrate into MB-GO dispersion, the mixture was stirred and refluxed for $2 \mathrm{~h}$ at $90{ }^{\circ} \mathrm{C}$. Then, the resulting dispersion was centrifuged and washed with deionized water for 3 times to obtain the corresponding water dispersible MB-rGO nanocomposite. The as-prepared MB-rGO was stored as aqueous dispersion of $1.0 \mathrm{mg} \mathrm{mL}^{-1}$.

The final content of $\mathrm{MB}$ in the nanocomposites was investigated by elemental analysis. The analysis results were $\mathrm{C}$ $71.86 \%$, H 2.863\%, N 8.120\% and S 3.927\%. Because nitrogen element may be introduced into the nanocomposites during the reduction process by hydrazine hydrate, the content of $\mathrm{MB}$ in the nanocomposites calculated based on the sulfur element was $39.17 \%$. The result indicated that enough MB molecules have been introduced in the nanocomposites.

\subsection{Preparation of MB-rGO/GC electrode}

Before the surface modification, the bare glassy carbon (GC) electrode with a diameter of $3.0 \mathrm{~mm}$ was polished with 1.0, 0.3, $0.05 \mu \mathrm{m}$ alumina powder on a polishing cloth, respectively, then cleaned under bath sonication for several minutes, and finally washed three times with deionized water. The cleaned GC electrode was dried with purified nitrogen stream. To prepare MB-rGO/GC electrode, $7 \mu \mathrm{L}$ MB-rGO aqueous dispersion $(1.0 \mathrm{mg}$ $\mathrm{mL}^{-1}$ ) was cast onto the GC electrode and dried in the air under an infrared lamp.

For comparison, $\mathrm{rGO} / \mathrm{GC}$ and $\mathrm{MB} / \mathrm{GC}$ electrodes were prepared by applying the same procedures as described above with rGO (1.0 mg mL $\left.\mathrm{mL}^{-1}\right)$ dispersion and $\mathrm{MB}\left(1.0 \mathrm{mg} \mathrm{mL}^{-1}\right)$ solution, respectively.

\subsection{Apparatus and measurements}

Transmission electron microscope (TEM, JEOL, Japan) characterization was carried out utilizing JEM-2100 with an accelerating voltage of $200 \mathrm{kV}$. Ultraviolet (UV)-visible experiments were performed with a Lambda $35 \mathrm{UV}$-vis spectrophotometer (Perkin Elmer Corp., USA). The Fourier transform infrared spectra (FT-IR) were recorded on a Spectrum One instrument (Perkin Elmer Corp., USA) by KBr-pellet. The zeta-potential values were obtained by using Zetasizer Nano-ZS particle analyzer (Malvern Corp., England). The morphologies of films were obtained by Hitachi SU-8010 scanning electron microscopy (SEM), equipped with an energy-dispersive spectroscopy (EDS) analysis system. X-ray diffraction (XRD) patterns were recorded using a D8 ADVANCE diffractometer (Bruker, Germany). Elemental analysis data were collected by Vario III elemental analyser (Elementar Corp., Germany). X-ray photoelectron spectroscopy (XPS) was conducted on an AXIS ULTRA spectrometer (Kratos Analytical Ltd.).

All electrochemical measurements were performed at room temperature by a BAS100B workstation (Bioanalytical Systems Inc., USA) in a conventional three-electrode system, using the as-prepared modified electrode as the working electrode, a platinum wire as the auxiliary electrode, and a saturated $\mathrm{Ag} /$ $\mathrm{AgCl}$ electrode as the reference electrode. All experiments were performed in $0.1 \mathrm{M}$ phosphate buffer solutions (PBS) at $\mathrm{pH}$ 7.4. The buffer solution was purged with highly purified nitrogen for at least $30 \mathrm{~min}$ and a nitrogen atmosphere environment was maintained during all electrochemical measurements without special statement. 


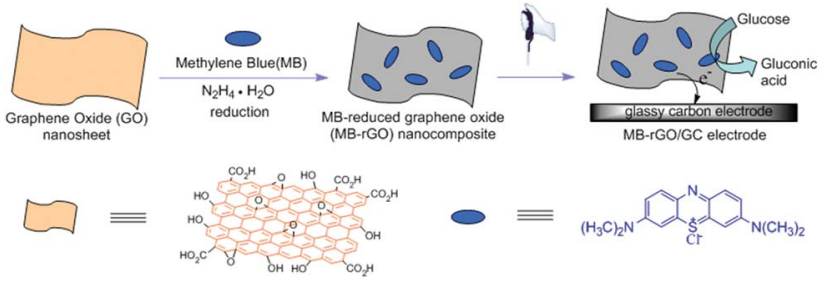

Scheme 1 Scheme of fabrication of MB-rGO/GC electrode.

\section{Results and discussion}

\subsection{Fabrication of MB-rGO/GC electrode}

The idealized scheme for design and construction of a MB-rGO/ GC electrode was depicted in Scheme 1. As shown, GO was firstly reduced by hydrazine in the presence of MB. Since the oxygen-functionalities of $\mathrm{GO}$ were removed, $\mathrm{sp}^{2}$-carbon hybrid structures were restored on the plane of rGO. In view of the surface of MB contained lots of $\pi$-bonds, MB could be adsorbed onto the surface of $\mathrm{rGO}$ via $\pi-\pi$ stacking interactions. As known, GO was directly reduced by hydrazine, which led to obviously irreversible aggregation in water. However, there was no precipitation in our reduction procedure, indicating that water-soluble $\mathrm{MB}$ played a crucial role in preventing the aggregation of rGO nanosheets. Being a cationic dye, the MB on the surface of rGO endowed the nanocomposite with positive charge, facilitating the dispersity of rGO via electrostatic repulsion. Finally, the MB-rGO/GC electrode was fabricated after MB-rGO aqueous dispersion cast onto the GC electrode.

\subsection{Characterization of MB-rGO nanocomposite}

The surface morphologies of the GO and MB-rGO nanocomposite were characterized by SEM. Fig. $1 \mathrm{~A}$ and B displayed the SEM images of GO and MB-rGO, respectively. As shown, the surface of MB-rGO was much fluctuant, compared to that of GO,
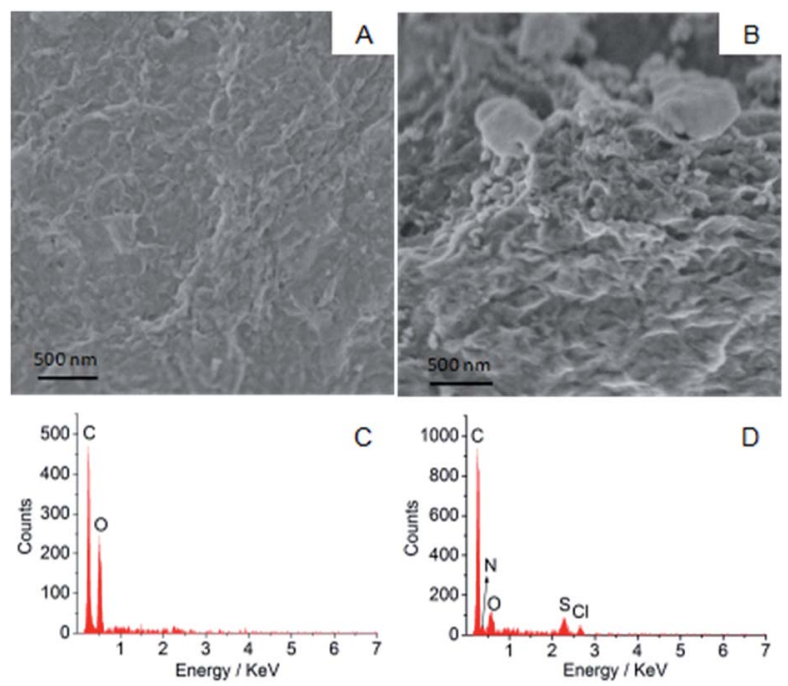

Fig. 1 SEM images of GO (A), MB-rGO (B) and EDS spectra of GO (C), MB-rGO (D). which was attributed to the modification of $\mathrm{MB}$ onto the surface of rGO. The EDS spectra of GO and MB-rGO nanocomposite were shown in Fig. 1C and D. As can be seen, GO contained C, O elements, however, MB-rGO contained the $\mathrm{C}, \mathrm{O}, \mathrm{N}, \mathrm{S}, \mathrm{Cl}$ elements. The $\mathrm{N}, \mathrm{S}, \mathrm{Cl}$ elements came from MB in the MB-rGO nanocomposite.

The TEM analysis was used to examine the morphological character of the MB-rGO nanocomposite. Fig. $2 \mathrm{~A}$ and B showed the typical TEM images of GO and MB-rGO nanocomposite, respectively. It could be observed that GO was in the form of corrugated sheet with partially curly edge, and the size of the sheets was micrometer scale. However, the morphology of MBrGO exhibited notable differences. Compared with GO sheets, the surface of MB-rGO was much rougher, indicating $\mathrm{MB}$ molecules were successfully modified onto $\mathrm{rGO}$, and the thickness of nanosheets was increased owning to the $\pi-\pi$ interactions of the conjugated molecular systems. The XRD spectra of graphite (a), GO (b), and MB-rGO (c) were shown in Fig. 2C. As shown, the peak of graphite at $26.5^{\circ}$ corresponded to the (002) reflection in the spectrum of graphite. However, the peak disappeared and a new peak appeared at $11.5^{\circ}(001)$ in spectrum of GO, indicating that graphite had been oxidized completely. As to the XRD spectrum of MB-rGO, the peak at $24.3^{\circ}(002)$ was weaker and wider than that of graphite, which was attributed to the effect of MB. Compared with the zeta potential value of $\mathrm{GO}(-40 \mathrm{mV}$, curve a in Fig. $2 \mathrm{D})$, the zeta potential value of MB-rGO (curve b in Fig. 2D) was about $+47.5 \mathrm{mV}$ under the same conditions. The positive charge of MB-rGO nanocomposite was ascribed to the introduction of MB. And, the nanocomposite displayed good dispersibility in water due to the sufficient electrostatic repulsion. The digital images of GO (c) and MB-rGO (d) dispersion were shown in the inset of Fig. 2D, respectively. As can be observed, owing to the existence of numerous hydrophilic functionalized groups on
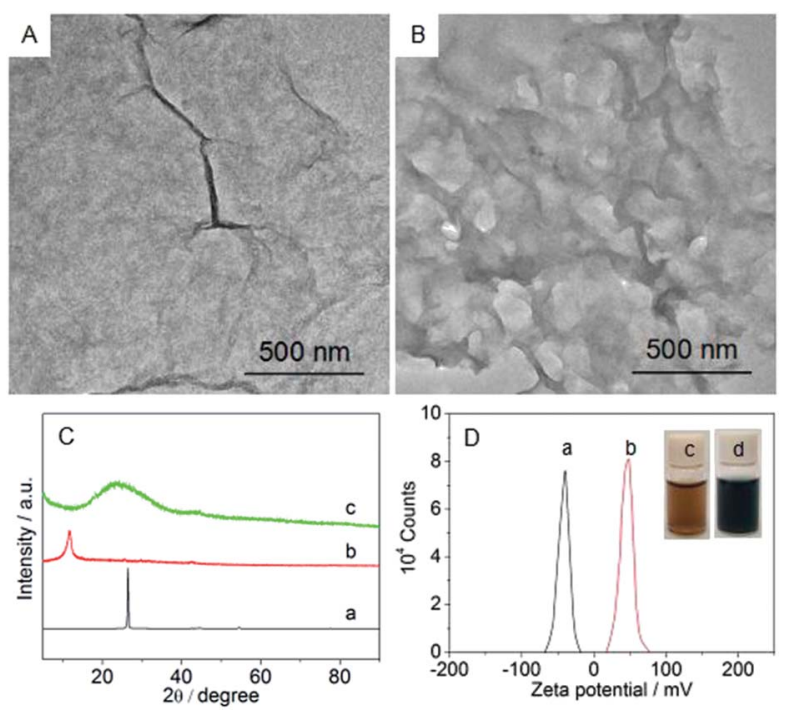

Fig. 2 TEM images of (A) GO, (B) MB-rGO; (C) XRD patterns of graphite (a), GO (b), MB-rGO (c), and (D) zeta-potential data of GO (a), MB-rGO (b) in water, inset: photographs of GO (c), MB-rGO (d) dispersed in water. 
the nanosheets, GO was readily dispersed in water and presented yellow-brown. By contrast, after the reduction of GO and modification by MB, the MB-rGO nanocomposite could be also well dispersed in water and exhibited a well dispersibility in water, and formed a homogeneous black-blue suspension. Even after standing for several weeks, the homogeneous suspension was still stable.

UV-vis spectroscopy was conducted to investigate the formation of MB-rGO nanostructure. Fig. 3A showed the UV-vis spectra of GO (a), MB (b) and MB-rGO (c) in aqueous solution, respectively. As shown, the spectrum of GO exhibited an absorption peak at $230 \mathrm{~nm}$ and a shoulder peak at $300 \mathrm{~nm}$, which was attributed to the $\pi-\pi$ transitions of aromatic $\mathrm{C}-\mathrm{C}$ bonds and the $n-\pi$ transitions of aromatic $\mathrm{C}=\mathrm{O}$ bonds, respectively. ${ }^{39}$ The spectrum of $\mathrm{MB}$ displayed two typical absorbance peaks at $291 \mathrm{~nm}$ and $663 \mathrm{~nm}$, whereas the shoulder peak at $245 \mathrm{~nm}$ and $621 \mathrm{~nm}$ were assigned to the absorbance peaks of the $\mathrm{MB}$ dimer in aqueous solution, which were consistent with the literature reported. ${ }^{40}$ However, after the formation of MB-rGO, the absorption peak redshifted to $268 \mathrm{~nm}$. As previously known, the rGO in water presented a strong absorption band at around $263 \mathrm{~nm} .{ }^{41}$ The slight redshift from 263 to $268 \mathrm{~nm}$ was attributed to $\pi-\pi$ interactions between MB and rGO, suggesting that the electronic conjugation structure within the MB-rGO nanocomposite was expanded after the self-assembly process. The FTIR spectra of GO (a), MB (b) and MB-rGO (c) were shown in Fig. 3B. From curve a, the
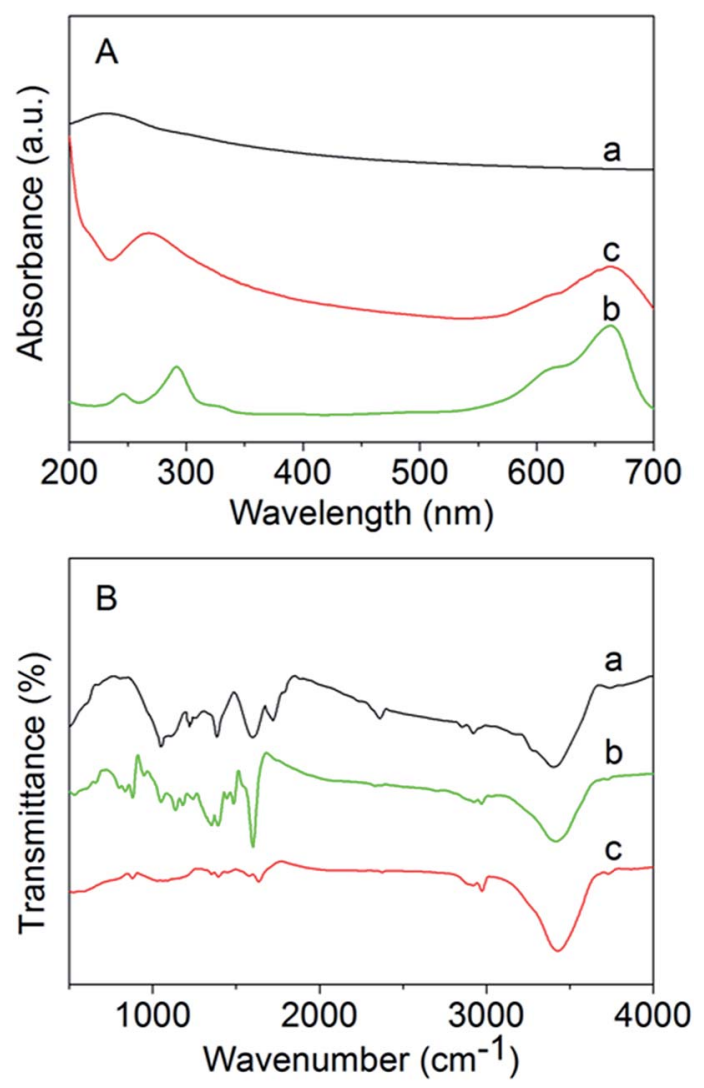

Fig. 3 (A) UV-vis adsorption spectra of $G O(a), M B(b)$ and MB-rGO (c) in water. (B) FTIR spectra of GO (a), MB (b) and MB-rGO (c). spectrum clearly exhibited a broad adsorption band at $3420 \mathrm{~cm}^{-1}$, which was assigned to the $\mathrm{OH}$ groups of GO. The other adsorption bands were shown the presence of $\mathrm{C}=\mathrm{O}(\nu \mathrm{C}=$ $\mathrm{O}$ at $\left.1720 \mathrm{~cm}^{-1}\right), \mathrm{C}-\mathrm{OH}\left(\nu \mathrm{C}-\mathrm{OH}\right.$ at $\left.1220 \mathrm{~cm}^{-1}\right)$ and $\mathrm{C}-\mathrm{O}(\nu \mathrm{C}-\mathrm{O}$ at $1052 \mathrm{~cm}^{-1}$ ) from carbonyl, carboxylic acid and alkoxy moieties, respectively. The spectrum of the pure MB displayed the presence of $\mathrm{C}-\mathrm{N}$ (at $1398 \mathrm{~cm}^{-1}$ ) and $-\mathrm{CH}_{3}\left(\right.$ at $\left.1354 \mathrm{~cm}^{-1}\right) .{ }^{42}$ However, the vibrational bands of oxygen-containing groups at 1720 and $1220 \mathrm{~cm}^{-1}$ almost disappeared and the peak at $1052 \mathrm{~cm}^{-1}$ markedly decreased in the spectrum of MB-rGO, suggesting that GO had been reduced to rGO. Additionally, the FTIR spectrum of MB-rGO still retained the peaks of $\mathrm{C}-\mathrm{N}$ and $-\mathrm{CH}_{3}$, which proved that the adsorption of $\mathrm{MB}$ onto the rGO was reliable. The above-mentioned results illustrated that the MB-rGO nanocomposite was successfully prepared.

The XPS spectra of the MB-rGO nanocomposite were characterized in Fig. S1A. $\uparrow$ As shown, the absorbance peaks at $284.1 \mathrm{eV}$ and $531.1 \mathrm{eV}$ were the $\mathrm{C}$ 1s and $\mathrm{O}$ 1s XPS spectra, respectively. ${ }^{43}$ Besides, the absorbance peaks of the $\mathrm{N} 1 \mathrm{~s}(400.0$ $\mathrm{eV}),{ }^{44} \mathrm{~S} 2 \mathrm{p}(163.6 \mathrm{eV})^{45}$ and $\mathrm{Cl} \mathrm{2p}(199.3 \mathrm{eV})^{46}$ also could be observed. These results illustrated that MB-rGO had been successfully prepared again. Specifically, for the C 1s spectra as shown in Fig. S1B, $\uparrow$ the absorbance peaks of the MB-rGO at 284.6, 285.7, 286.5, 287.7, 289.0 eV were $\mathrm{C}-\mathrm{C}, \mathrm{C}-\mathrm{N}, \mathrm{C}-\mathrm{O}, \mathrm{C}=\mathrm{O}$, $\mathrm{O}-\mathrm{C}=\mathrm{O}$, respectively, ${ }^{47}$ and the peak of $\mathrm{C}=\mathrm{O}$ and $\mathrm{O}-\mathrm{C}=\mathrm{O}$ were small, indicating that GO was commendably reduced. The peaks of $\mathrm{N} 1 \mathrm{~s}$ located at 398.2 and $400.8 \mathrm{eV}$ (Fig. S1C $\dagger$ ), assigned to pyridinic nitrogen and graphitic nitrogen, respectively. ${ }^{48}$ The main peaks of $\mathrm{O} 1 \mathrm{~s}$ at 533.2 and $531.6 \mathrm{eV}$ in Fig. $\mathrm{S} 1 \mathrm{D} \dagger$ were associated with $\mathrm{C}-\mathrm{O}$ and carbonyl group $(\mathrm{C}=\mathrm{O})$, respectively. ${ }^{49}$ Fig. S1E† showed S 2p XPS spectrum, which was spin-orbit splitting of $S 2 p$ peak, indicating there was a state of sulfur. ${ }^{50}$ The peak of $\mathrm{Cl} 2 \mathrm{p}$ at $198.7 \mathrm{eV}$ (Fig. S1F $\dagger$ ) indicated that MB-rGO nanocomposite included the $\mathrm{Cl}^{-}$counter ions. ${ }^{51}$

\subsection{Electrochemical properties of MB-rGO/GC electrode}

To investigate the effect of immobilized amount of MB-rGO modified electrode on the redox currents, different concentrations of MB-rGO dispersion were cast on the surface of GC electrode. The cyclic voltammograms (CVs) of MB-rGO electrode were shown in the Fig. 4, the peak current of MB-rGO/GC electrode increased with the increasement of concentration from 0.1 to $2.0 \mathrm{mg} \mathrm{mL}{ }^{-1}$. When the concentration was greater than $1.0 \mathrm{mg} \mathrm{mL}^{-1}$, the peak current of MB-rGO/GC electrode was no obvious change. The results demonstrated that the thickness of composite film would affect the following glucose sensing performance. And the GC electrode prepared with $1.0 \mathrm{mg} \mathrm{mL}^{-1}$ of MB-rGO dispersion was finally selected in the subsequent experiments.

The CVs of bare GC, rGO/GC, MB/GC and MB-rGO/GC electrodes were shown in Fig. 5 and no redox peaks were observed at bare GC (Fig. 5a) and rGO/GC (Fig. 5b), indicating rGO was not electroactive in the potential range. However, a pair of asymmetric redox peaks were observable at the formal potential $\left(E_{\mathrm{p}}\right)$ of $-0.203 \mathrm{~V}$ at the MB/GC electrode (Fig. 5c). For the MB-rGO/ GC electrode, a couple of nearly symmetric and well-defined 


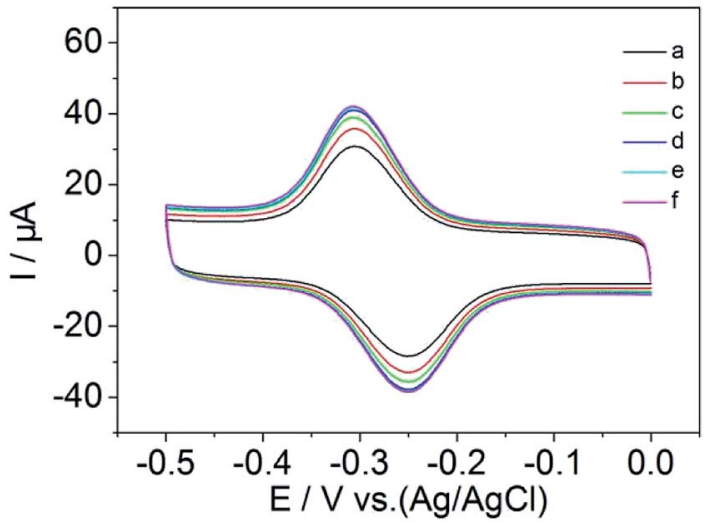

Fig. 4 Cyclic voltammograms of the MB-rGO/GC electrode with casting 0.1 (a), 0.2 (b), 0.5 (c), 1.0 (d), 1.5 (e), 2.0 (f) $\mathrm{mg} \mathrm{mL}^{-1}$ of MB-rGO dispersion in 0.1 M PBS ( $\mathrm{pH}$ 7.4). Scan rate, $200 \mathrm{mV} \mathrm{s}^{-1}$.

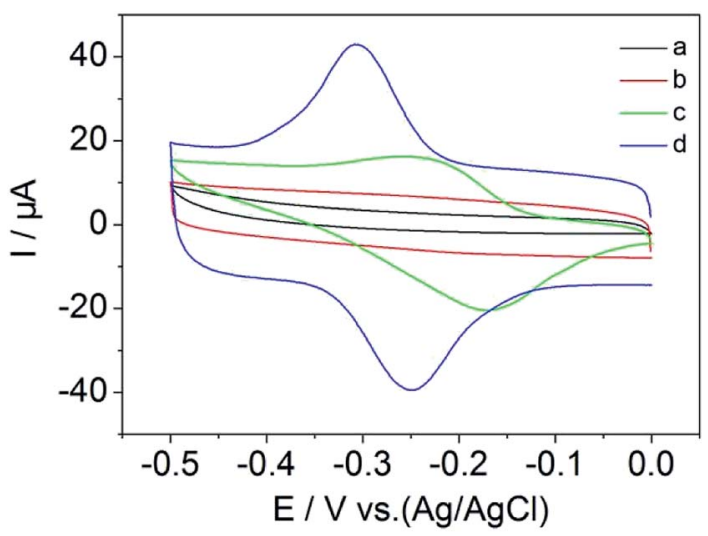

Fig. 5 Cyclic voltammograms of bare GC (a), rGO/GC (b), MB/GC (c) and MB-rGO/GC (d) electrodes in $0.1 \mathrm{M}$ PBS (pH 7.4). Scan rate, $200 \mathrm{mV} \mathrm{s}^{-1}$.

redox peaks were observed at MB-rGO/GC electrode (Fig. 5d) in the same potential range. The redox peak potentials were -0.308 and $-0.254 \mathrm{~V}$, respectively. The $E_{\mathrm{p}}$ calculated from the average of the cathodic and anodic peak potentials was $-0.281 \mathrm{~V}$, which was close to the previous literature, ${ }^{38}$ illustrating that rGO facilitated electron transfer between $\mathrm{MB}$ and GC electrode. The peak-to-peak separation of cathodic and anodic peak was $54 \mathrm{mV}$ and the ratio of oxidation and reduction peak currents approximated to 1 , demonstrating the quasireversible fast electron transfer of a redox reaction at the electrode. The above results indicated that MB-rGO nanocomposite possessed excellent electron-transport properties, which could realize the fast electron transfer between MB-rGO and the GC electrode.

The effect of the scan rate on the response of MB-rGO/GC electrode and the plots of peak currents versus scan rate were depicted in Fig. 6. As shown, the cathodic peak potential shifted to a more negative value with an increasing scan rate, while the anodic peak potential shifted in a positive direction. And it is obvious that both the reduction and oxidation peak currents $\left(I_{\mathrm{p}}\right)$ were gradually increased with the increment of scan rate from
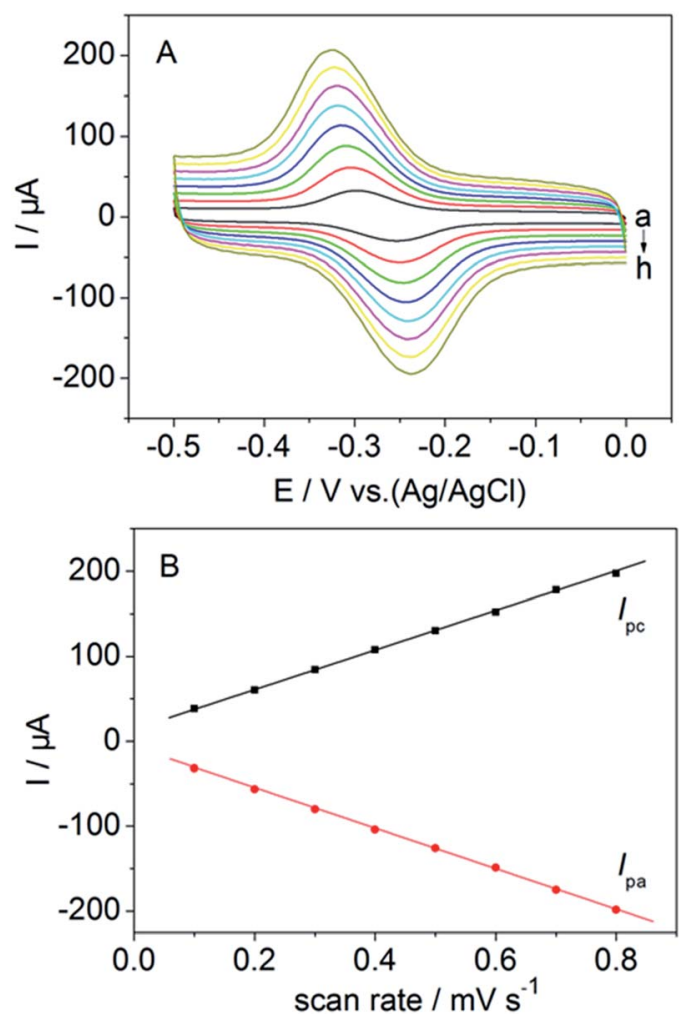

Fig. 6 (A) Cyclic voltammograms of the MB-rGO/GC electrode at scan rates of 100, 200, 300,400,500, 600, 700 and $800 \mathrm{mV} \mathrm{s}^{-1}$ (from (a) to (h)) in 0.1 M PBS (pH 7.4), respectively. (B) Plots of the oxidation peak current $(\mathbf{O})$ and reduction peak current $(\mathbf{\square})$ versus scan rate for the MB-rGO/GC electrode.

100 to $800 \mathrm{mV} \mathrm{s}^{-1}$. This demonstrated that the electrochemical process at the MB-rGO/GC electrode is a surface-controlled process with a fast electron transfer. ${ }^{52}$

\subsection{Electrocatalytic properties of MB-rGO/GC electrode}

The performance of MB-rGO/GC electrode-based glucose sensor was investigated by CVs and differential pulse voltammograms (DPVs) techniques. Fig. 7 showed the CVs of MB-rGO/GC electrode in the deoxygenated (curve a) and $\mathrm{O}_{2}$-saturated $\mathrm{PBS}$ in the absence (curve b) and presence (curve c) of glucose. As aforementioned, curve a (the same as Fig. 5d) displayed a pair of symmetrical redox peaks in $\mathrm{N}_{2}$-saturated PBS. By comparison, the reduction current of the $\mathrm{MB}-\mathrm{rGO} / \mathrm{GC}$ electrode in curve b dramatically increased under saturated $\mathrm{O}_{2}$ condition, accompanied by a decrease of oxidation current. Furthermore, when glucose was added into $\mathrm{O}_{2}$-saturated PBS, the reduction peak current decreased (curve c). This was due to the consumption of dissolved oxygen at electrode surface by glucose, resulting in decrease of the concentration of the oxidized form of MB.

Fig. 8 displayed the electrocatalytic activity of the MB-rGO/ GC electrode. The DPVs of the MB-rGO/GC electrode in the $\mathrm{O}_{2}$-saturated PBS containing different glucose concentrations was shown in Fig. 8A. The reduction current at $-0.28 \mathrm{~V}$ decreased gradually with the increment of glucose 


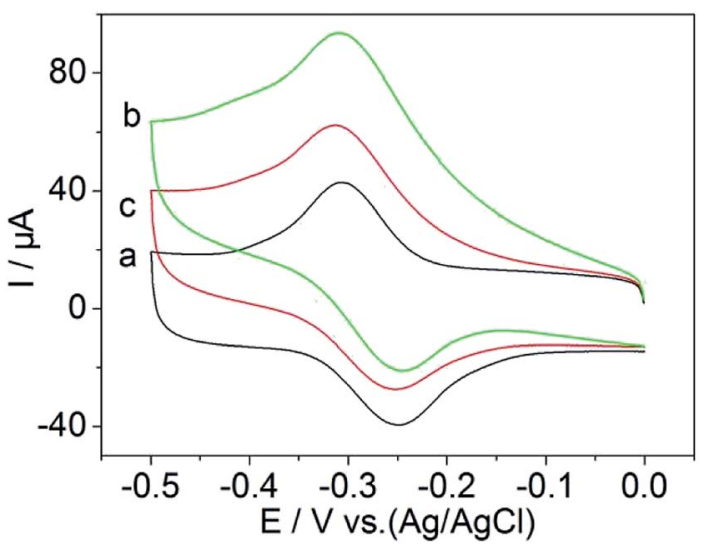

Fig. 7 Cyclic voltammograms of the MB-rGO/GC electrode in the $\mathrm{N}_{2}-$ saturated PBS (a), in the $\mathrm{O}_{2}$-saturated PBS (b), and in the $\mathrm{O}_{2}$-saturated PBS after addition of glucose (final concentration of $11.44 \mathrm{mM}$ ) (c), scan rate: $200 \mathrm{mVs}^{-1}$.
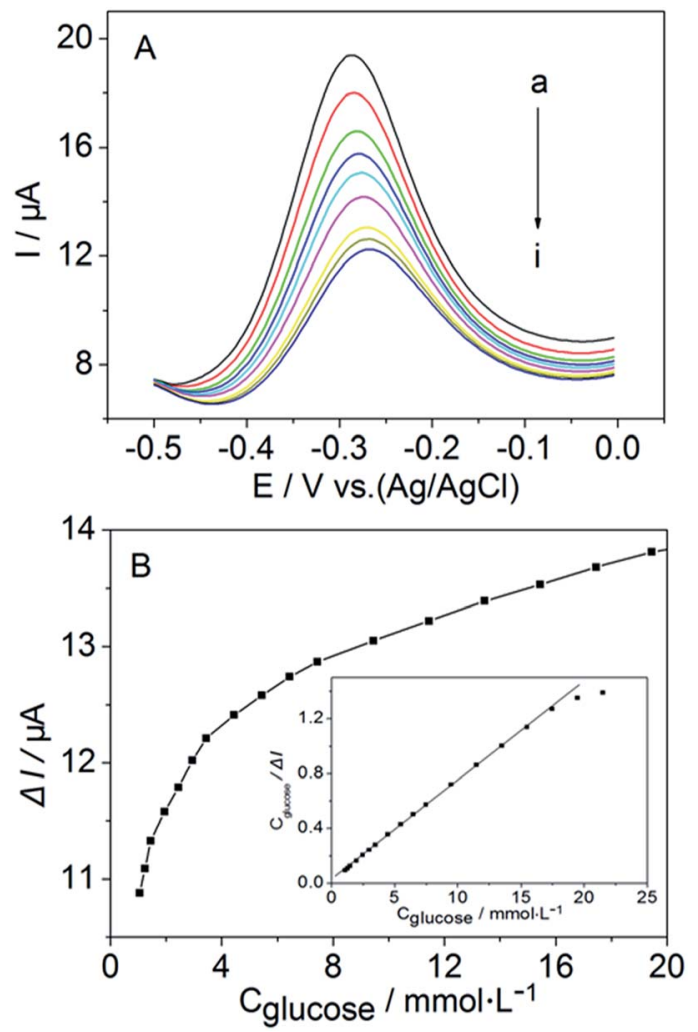

Fig. 8 (A) DPVs of MB-rGO/GC electrode in $0.1 \mathrm{M} \mathrm{O}_{2}$-saturated PBS (pH 7.4) with (a) 0, (b) 1.04, (c) 1.44, (d) 1.94, (e) 2.94, (f) 4.44, (g) 6.44, (h) 9.44, (i) $13.44 \mathrm{mM}$ glucose, respectively. (B) Plot of the catalytic current $(\Delta /)$ versus glucose concentration for the MB-rGO/GC electrode. Inset: plot of the glucose concentration/catalytic current $(C / \Delta /)$ versus glucose concentration for the MB-rGO/GC electrode. Amplitude: $50 \mathrm{mV}$; pulse width: $50 \mathrm{~ms}$.

concentration in the PBS, which indicated that the MB-rGO/GC electrode was active to oxidation of glucose. The electrocatalytic process of glucose by MB-rGO nanocomposite may be described by the following equations eqn (1)-(3).53,54

$$
\begin{gathered}
\mathrm{MB}^{+}+2 \mathrm{e}^{-}+\mathrm{H}^{+} \leftrightarrow \mathrm{MBH} \\
\mathrm{O}_{2}+2 \mathrm{MBH} \rightarrow 2 \mathrm{MB}^{+}+2 \mathrm{OH}^{-} \\
\mathrm{GL}+\mathrm{MB}^{+}+\mathrm{OH}^{-} \rightarrow \mathrm{MBH}+\mathrm{GLA}
\end{gathered}
$$

Where, $\mathrm{MBH}$ and $\mathrm{MB}^{+}$are the reduced and oxidized forms of methylene blue, respectively. GL is glucose, GLA is gluconic acid, and the rate of eqn (2) is much greater than the rate of eqn (3). Based on above analysis, the catalytic mechanism here is similar to that of GOx according to the literature reported, ${ }^{5,56}$ which illustrated that MB could act as GOx-mimicking for the homologous catalytic reaction. Fig. 8B depicted the relation between the decrement in reduction peak current of MB-rGO/ GC electrode and the concentration of glucose. It can be observed from the inset of Fig. 8B, the glucose concentration/ catalytic current increased linearly with the concentration ranges of glucose from 1.04 to $17.44 \mathrm{mM}$. The detection limit for the sensor was $45.8 \mu \mathrm{M}$, based on a signal-to-noise ratio of 3 . The wide linear range and low detection limit of the MB-rGO/GC electrode provide promising applications in glucose sensing. The linear regression equation was $y=0.072 x+0.031(R=$ 0.9997, $n=16$ ), in which $y$ and $x$ stand for the glucose concentration/catalytic current and the concentration (mM) of glucose, respectively. According to the slope of the line, the sensitivity of the MB-rGO/GC electrode was calculated to be $13.08 \mu \mathrm{A} \mathrm{mM}^{-1} \mathrm{~cm}^{-2}$, which was higher than that of the most other non-enzymatic glucose sensors previously reported (Table 1). As known, the normal human blood glucose concentration ranges from 4 to $7 \mathrm{mM},{ }^{64}$ and the glucose concentration of the diabetic is more than above-mentioned upper limit. Hence, the MB-rGO/GC electrode is suitable for the glucose detection in serum.

To further investigate the catalytic process, catalytic products were monitored by UV-vis adsorption spectra. According to the reported literature, ${ }^{65}$ gluconic acid can be indirectly detected by reacting with hydroxylamine-ferric chloride to form hydroxamate $\mathrm{Fe}^{3+}$ complex. So, the resulting of gluconic acid in this work can be detected by this method. As a comparison, gluconic acid obtained by the electrochemical catalytic reaction of GOx using GOx/Polymeric Ionic Liquids (PILs)-GP/GC electrode, which was reported by us, ${ }^{9}$ was also determined by the above method. Fig. 9 showed the UV-vis spectra of purchased glucose solution (curve a), the resulting solutions of MB-rGO/ GC electrode based system (curve b) and GOx/PILs-GP/GC electrode based system (curve c), respectively. As shown, there was no absorption peaks in the glucose solution, and the absorption peak of curve $\mathrm{b}$ appeared at $505 \mathrm{~nm},{ }^{66}$ which was similar to curve $\mathrm{c}$, suggesting that gluconic acid was produced in the MB-rGO system.

\subsection{Reproducibility and stability assay results}

To investigate the reproducibility of MB-rGO/GC electrode, five different electrodes, prepared independently with the same procedures, showed a good reproducibility with a relative standard deviation of $4.4 \%$ in the response to $5 \mathrm{mM}$ glucose. The stability of MB-rGO/GC electrode was also evaluated. On the 
Table 1 Comparison of the performance of our proposed MB-rGO sensor with other nonenzymatic glucose sensors

\begin{tabular}{|c|c|c|c|c|}
\hline Electrode material & Linear range $(\mathrm{mM})$ & Detection limit $(\mu \mathrm{M})$ & $\begin{array}{l}\text { Sensitivity } \\
\left(\mu \mathrm{A} \mathrm{mM}^{-1} \mathrm{~cm}^{-2}\right)\end{array}$ & Ref. \\
\hline $\mathrm{MWCNT}^{a} / \mathrm{NiO}$ & $0.2-12$ & 160 & - & 57 \\
\hline Pt NPs/MWCNT & $1.0-8.0$ & 50 & 11.83 & 59 \\
\hline Pt nanoflowers & $1-16$ & 48 & 1.87 & 60 \\
\hline $\mathrm{CoPcTS}^{c} / \mathrm{OPPyNF}^{d}$ & $0.25-20$ & 100 & 5.69 & 61 \\
\hline MB-rGO & $1.04-17.44$ & 45.8 & 13.08 & This work \\
\hline
\end{tabular}

one hand, after continuously scanning for 100 cycles, the peak current almost remained $92 \%$ of the initial response. On the other hand, the CVs were performed after the modified electrode was stored in $\mathrm{pH} 7.4$ buffers for a period of time. The variation of the reduction peak current was negligible after $8 \mathrm{~h}$, indicating that the MB-rGO/GC electrode possessed an acceptable stability. Additionally, the long-term stability of the MB$\mathrm{rGO} / \mathrm{GC}$ electrode was examined by measuring the sensor response in the presence of $5 \mathrm{mM}$ of glucose for one-month (Fig. S2 $\dagger$ ). As can be seen, the response current of the MB$\mathrm{rGO} / \mathrm{GC}$ electrode towards glucose decreased about $2.92 \%$ after first five days. After being stored for 15 days, the decrement of response current was $6.14 \%$ of its initial response, and the response current only decreased $7.98 \%$ after 30 days. Hence, the above-mentioned results indicated that the glucose sensor exhibited the suitable stability toward the electrocatalytic oxidation of glucose.

\subsection{Interference study and real sample assay}

Dopamine (DA), ascorbic acid (AA) and uric acid (UA) in serum are electrochemically active, which could be readily oxidized and consequently interfered the determination of glucose. ${ }^{11} \mathrm{As}$ can be seen in Fig. 10, the peak current obviously decreased

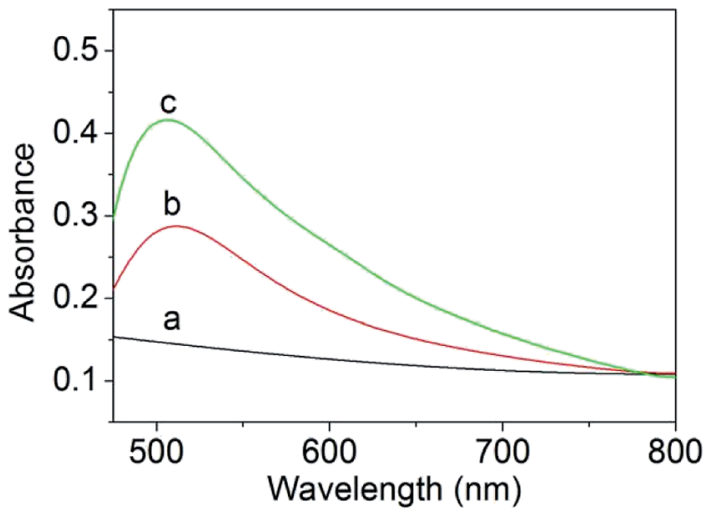

Fig. 9 Gluconic acid-specific colorimetric assay after the catalytic reaction. Purchased glucose solution (curve a), the resulting solutions of glucose oxidized by (curve b) and the GOx/PILs-GP/GC electrode ${ }^{9}$ (curve c).

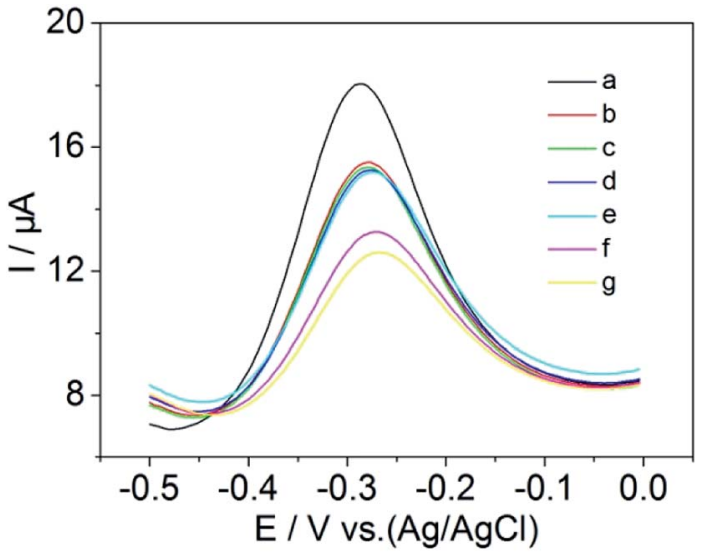

Fig. 10 DPVs of the MB-rGO/GC electrode in $0.1 \mathrm{M} \mathrm{O}_{2}$-saturated PBS $(\mathrm{pH} 7.4)$ in the presence of $1.04 \mathrm{mM}$ glucose (a), $2.44 \mathrm{mM}$ glucose (b), $0.1 \mathrm{mM}$ DA (c), $0.1 \mathrm{mM} \mathrm{AA}(\mathrm{d}), 0.1 \mathrm{mM} \cup \mathrm{A}(\mathrm{e}), 6.44 \mathrm{mM}$ glucose (f) and $13.44 \mathrm{mM}$ glucose $(\mathrm{g})$ in cyclic voltammetry measurements using MB$\mathrm{rGO} / \mathrm{GC}$ electrode.

with the increasement of glucose concentration. However, no obvious changes of peak current were caused after the addition of $0.1 \mathrm{mM} \mathrm{DA}, 0.1 \mathrm{mM} \mathrm{AA}$, and $0.1 \mathrm{mM} \mathrm{UA}$, which confirmed the high selectivity of the prepared glucose sensor.

In order to evaluate the feasibility of the proposed glucose sensor for real sample analysis. The sensor was used for the determination of glucose by standard addition methods in serum samples. The results are listed in Table 2. It can be found that the experimental results of glucose concentration in serum samples agreed well with the values obtained from glucometer,

Table 2 The results of glucose determination in human serum samples

\begin{tabular}{lllll}
\hline Sample & $\begin{array}{l}\text { Added } \\
(\mathrm{mM})\end{array}$ & $\begin{array}{l}\text { Found } \\
(\mathrm{mM})\end{array}$ & $\begin{array}{l}\text { Recovery } \\
(\%)\end{array}$ & $\begin{array}{l}\text { Hospital } \\
\text { data }(\mathrm{mM})\end{array}$ \\
\hline 1 & - & $5.11 \pm 0.08$ & - & 5.15 \\
& 0.3 & $5.42 \pm 0.13$ & 103.3 & \\
2 & 0.6 & $5.69 \pm 0.12$ & 96.7 & \\
& - & $5.84 \pm 0.17$ & - & 5.94 \\
& 0.3 & $6.12 \pm 0.11$ & 93.3 & \\
& 0.6 & $6.39 \pm 0.05$ & 91.7 &
\end{tabular}


and the recoveries for the determination of glucose were between $91 \%$ and $104 \%$, indicating the potential application of the proposed glucose sensor in clinical analysis.

\section{Conclusions}

In summary, a well-dispersed and good electroconductive MBrGO nanocomposite was prepared based on the $\pi-\pi$ stacking interactions. The as-prepared MB-rGO nanocomposite was further used to construct enzyme-free electrochemical glucose sensor. Electrochemical investigation indicated that the MBrGO modified electrode possessed glucose oxidase-mimetic activity and exhibited good electrocatalytic performance towards glucose with a wide linear range from 1.04 to $17.44 \mathrm{mM}$, a low detection limit of $45.8 \mu \mathrm{M}$ and a large sensitivity of 13.08 $\mu \mathrm{A} \mathrm{cm} \mathrm{cm}^{-2} \mathrm{mM}^{-1}$. The prepared glucose sensor exhibited high stability, reproducibility and good anti-interference ability. This research illustrates that $\mathrm{MB}$ is a promising redox-active molecule of electrode materials for developing enzyme-mimetic electrochemical sensors.

\section{Conflicts of interest}

There are no conflicts to declare.

\section{Acknowledgements}

This work was financially supported by the National Natural Science Foundation of China (No. 51773085), Liaoning Province Doctor Start-up Fund (No. 20170520282, 201501129), The Training Programs of Innovation and Entrepreneurship for Undergraduates of Liaoning University (No. x201710140198) and Youth Fund of Liaoning University (No. LDQN2015006).

\section{Notes and references}

1 G. Danaei, M. M. Finucane, Y. Lu, G. M. Singh, M. J. Cowan, C. J. Paciorek, J. K. Lin, F. Farzadfar, Y.-H. Khang, G. A. Stevens, M. Rao, M. K. Ali, L. M. Riley, C. A. Robinson and M. Ezzati, Global Burden of Metabolic Risk Factors of Chronic Diseases Collaborating Group (Blood Glucose), Lancet, 2011, 378, 31-40.

2 G. Zhao, X. Hou, X. Li, M. Qu, C. Tong and W. Li, Int. J. Biol. Macromol., 2018, 108, 550-557.

3 K. R. Ahmed, F. Jebunessa, S. Hossain and H. A. Chowdhury, BMC Ophthalmol., 2017, 17, 171.

4 S. Wild, G. Roglic, A. Green, R. Sicree and H. King, Diabetes Care, 2004, 27, 1047-1053.

5 J. M. Yuen, N. C. Shah, J. T. Walsh Jr, M. R. Glucksberg and R. P. Van Duyne, Anal. Chem., 2010, 82, 8382-8385.

6 Y. Gao, Y. Wu and J. Di, Spectrochim. Acta, Part A, 2017, 173, 207-212.

7 S. Mittelmaier, M. Fünfrocken, D. Fenn, T. Fichert and M. Pischetsrieder, J. Chromatogr. B: Anal. Technol. Biomed. Life Sci., 2010, 878, 877-882.

8 J. Liu, C. Guo, C. M. Li, Y. Li, Q. Chi, X. Huang, L. Liao and T. Yu, Electrochem. Commun., 2009, 11, 202-205.
9 Q. Zhang, S. Wu, L. Zhang, J. Lu, F. Verproot, Y. Liu, Z. Xing, J. Li and X.-M. Song, Biosens. Bioelectron., 2011, 26, 26322637.

10 Y. Liu, H. Teng, H. Hou and T. You, Biosens. Bioelectron., 2009, 24, 3329-3334.

11 B. Wang, Y. Wu, Y. Chen, B. Weng and C. Li, Sens. Actuators, $B, 2017,238,802-808$.

12 X. Xiao, B. Zhou, L. Zhu, L. Xu, L. Tan, H. Tang, Y. Zhang, Q. Xie and S. Yao, Sens. Actuators, B, 2012, 165, 126-132.

13 J. Yang, W. Tan, C. Chen, Y. Tao, Y. Qin and Y. Kong, Mater. Sci. Eng., C, 2017, 78, 210-217.

14 H.-C. Wang and A.-R. Lee, J. Food Drug Anal., 2015, 23, 191200.

15 M. M. Rahman, A. J. S. Ahammad, J. H. Jin, J. A. Sang and J. J. Lee, Sensors, 2010, 10, 4855-4886.

16 S. Y. Tee, C. P. Teng and E. Ye, Mater. Sci. Eng., C, 2017, 70, 1018-1030.

17 S. Park, H. Boo and T. D. Chung, Anal. Chim. Acta, 2006, 556, 46-57.

18 T. M. Cheng, T. K. Huang, H. K. Lin, S. P. Tung, Y. L. Chen, C. Y. Lee and H. T. Chiu, ACS Appl. Mater. Interfaces, 2010, 2, 2773.

19 J. H. Yuan, K. Wang and X. H. Xia, Adv. Funct. Mater., 2010, 15, 803-809.

20 F. Cao and J. Gong, Anal. Chim. Acta, 2012, 723, 39-44.

21 C. Guo, Y. Wang, Y. Zhao and C. Xu, Anal. Methods, 2013, 5, 1644-1647.

22 Q. N. Dung, D. Patil, H. Jung and D. Kim, Biosens. Bioelectron., 2013, 42, 280-286.

23 M. Liu, R. Liu and W. Chen, Biosens. Bioelectron., 2013, 45, 206-212.

24 K. Bamba, J. M. Léger, E. Garnier, C. Bachmann, K. Servat and K. B. Kokoh, Electrochim. Acta, 2005, 50, 3341-3346.

25 L. M. Santos and R. P. Baldwin, Anal. Chem., 1987, 59, 17661770.

26 C. Wang, Y. Sun, X. Yu, D. Ma, J. Zheng, P. Dou, Z. Cao and X. Xu, J. Mater. Sci.: Mater. Electron., 2016, 27, 9370-9378.

27 Y. B. Vassilyev, O. A. Khazova and N. N. Nikolaeva, J. Electroanal. Chem. Interfacial Electrochem., 1985, 196, 105125.

28 G. Dutta and P. B. Lillehoj, Analyst, 2017, 142, 3492-3499.

29 H.-A. Rafiee-Pour, M. Behpour and M. Keshavarz, Biosens. Bioelectron., 2016, 77, 202-207.

30 X. Lin, Y. Ni and S. Kokot, Anal. Chim. Acta, 2015, 867, 29-37. 31 Q. Zhang, S. Yang, J. Zhang, L. Zhang, P. Kang, J. Li, J. Xu, H. Zhou and X.-M. Song, Nanotechnology, 2011, 22, 494010. 32 Q. Zhang, X. Lv, Y. Qiao, L. Zhang, D. L. Liu, W. Zhang, G. X. Han and X.-M. Song, Electroanalysis, 2010, 22, 10001004.

33 Y. Yan, M. Zhang, K. Gong, L. Su, Z. Guo and L. Mao, Chem. Mater., 2005, 17, 3457-3463.

34 Q. Zhang, Y. Qiao, F. Hao, L. Zhang, S. Wu, Y. Li, J. Li and X.-M. Song, Chem.-Eur. J., 2010, 16, 8133-8139.

35 Q. Zhang, Y. Qiao, L. Zhang, S. Wu, H. Zhou, J. Xu and X.-M. Song, Electroanalysis, 2011, 23, 900-906.

36 X. Wang, J. Wang, H. Cheng, P. Yu, J. Ye and L. Mao, Langmuir, 2011, 27, 11180-11186. 
37 S. Wu, Y. Wang, H. Mao, C. Wang, L. Xia, Y. Zhang, H. Ge and X.-M. Song, RSC Adv., 2016, 6, 59487-59496.

38 Z. Li, X.-J. Shi, X.-P. Ge, J.-J. Wei, C.-Z. Yang, B. Fang, H.-F. Xie and X.-C. An, Chem. Res. Chin. Univ., 2012, 28, 520-523.

39 C. Zhang, L. Ren, X. Wang and T. Liu, J. Phys. Chem. C, 2010, 114, 11435-11440.

40 Q. Han, Y. Wang, Y. Huang, L. Guo and Y. Fu, Analyst, 2013, 138, 2051-2056.

41 H. Liu, J. Gao, M. Xue, N. Zhu, M. Zhang and T. Cao, Langmuir, 2009, 25, 12006-12010.

42 D. Zhang, L. Fu, L. Liao, B. Dai, R. Zou and C. Zhang, Electrochim. Acta, 2012, 75, 71-79.

43 J. Yang, W. Tan, C. Chen, Y. Tao, Y. Qin and Y. Kong, Mater. Sci. Eng., C, 2017, 78, 210-217.

44 F. Li, J. Chai, H. Yang, D. Han and L. Niu, Talanta, 2010, 81, 1063-1068.

45 J. Li, X. Rao, F. Xiang, J. Wei, M. Yuan and Z. Liu, Analyst, 2018, 143, 2083-2089.

46 P. Gawali and B. L. Jadhav, Process Biochem., 2018, 69, 106122.

47 C. Shan, H. Yang, J. Song, D. Han, A. Ivaska and L. Niu, Anal. Chem., 2009, 81, 2378-2382.

48 Z.-H. Sheng, L. Shao, J.-J. Chen, W.-J. Bao, F.-B. Wang and X.-H. Xia, ACS Nano, 2011, 5, 4350-4358.

49 S. Chebil, M. O. Monod and P. Fisicaro, Electrochim. Acta, 2014, 123, 527-534.

50 X. Yang, L. Xiong, X. Hu, B. He and G. Chu, Res. Chem. Intermed., 2012, 38, 67-75.

51 Y.-K. Yang, C.-E. He, R.-G. Peng, A. Baji, X.-S. Du, Y.-L. Huang, X.-L. Xie and Y.-W. Mai, J. Mater. Chem., 2012, 22, 5666-5675.
52 A. J. Brad and L. R. Faulkner, Electrochemical methods: Fundamentals and applications, John Wiley \& Sons, Inc, 2nd edn, 2001.

53 G. A. Evtugyn, V. B. Stepanova, A. V. Porfireva, A. I. Zamaleeva and R. R. Fakhrullin, J. Nanosci. Nanotechnol., 2014, 14, 6738-6747.

54 A. J. Pons, F. Sagués, M. A. Bees and P. G. Sørensen, J. Phys. Chem. B, 2000, 104, 2251-2259.

55 S. Liu and H. Ju, Biosens. Bioelectron., 2003, 19, 177-183.

56 C. Deng, J. Chen, X. Chen, C. Xiao, L. Nie and S. Yao, Biosens. Bioelectron., 2008, 23, 1272-1277.

57 M. Shamsipur, M. Najafi and M.-R. M. Hosseini, Bioelectrochemistry, 2010, 77, 120-124.

58 L. Meng, Y. Xia, W. Liu, L. Zhang, P. Zou and Y. Zhang, Electrochim. Acta, 2015, 152, 330-337.

59 L.-H. Li and W.-D. Zhang, Microchim. Acta, 2008, 163, 305311.

60 M. Q. Guo, H. S. Hong, X. N. Tang, H. D. Fang and X. H. Xu, Electrochim. Acta, 2012, 63, 1-8.

61 L. Özcan, Y. Şahin and H. Türk, Biosens. Bioelectron., 2008, 24, 512-517.

62 N. Hui, W. Wang, G. Xu and X. Luo, J. Mater. Chem. B, 2015, 3, 556-561.

63 S. Liu, Y. Ma, R. Zhang and X. Luo, ChemElectroChem, 2016, 3, 1799-1804.

64 T. Dayakar, R. K. Venkateswara, k. M. Vinod, K. Bikshalu, B. Chakradhar and R. K. Ramachandra, Appl. Surf. Sci., 2017, 435, 216-224.

65 Y. Zhao, Y. Huang, H. Zhu, Q. Zhu and Y. Xia, J. Am. Chem. Soc., 2016, 138, 16645-16654.

66 E. T. Rakitzis and P. Papandreou, Chem.-Biol. Interact., 1998, 113, 205-216. 